\title{
Theory of elastic and piezoelectric effects in two-dimensional hexagonal boron nitride
}

\author{
K. H. Michel and B. Verberck \\ Departement Fysica, Universiteit Antwerpen, Groenenborgerlaan 171, 2020 Antwerpen, Belgium \\ (Received 6 October 2009; revised manuscript received 20 November 2009; published 17 December 2009)
}

\begin{abstract}
Starting from an empirical force constant model of valence interactions and calculating by Ewald's method the ion-ion force constants, we derive the dynamical matrix for a monolayer crystal of hexagonal boron nitride (h-BN). The phonon dispersion relations are calculated. The interplay between valence and Coulomb forces is discussed. It is shown by analytical methods that the longitudinal and the transverse optical (LO and TO) phonon branches for in-plane motion are degenerate at the $\Gamma$ point of the Brillouin zone. Away from $\Gamma$, the LO branch exhibits pronounced overbending. It is found that the nonanalytic Coulomb contribution to the dynamical matrix causes a linear increase of the LO branch with increasing wave vector starting at $\Gamma$. This effect is general for two-dimensional (2D) ionic crystals. Performing a long-wavelength expansion of the dynamical matrix, we use Born's perturbation method to calculate the elastic constants (tension coefficients). Since the crystal is noncentrosymmetric, internal displacements due to relative shifts between the two sublattices (B and $\mathrm{N})$ contribute to the elastic constants. These internal displacements are responsible for piezoelectric and dielectric phenomena. The piezoelectric stress constant and the dielectric susceptibility of $2 \mathrm{D}$ h-BN are calculated.
\end{abstract}

DOI: 10.1103/PhysRevB.80.224301

PACS number(s): 61.46.- w, 62.20.-x, 68.65.-k, 77.65.- $\mathrm{j}$

\section{INTRODUCTION}

The experimental discovery of graphene and other freestanding two-dimensional (2D) crystals $^{1,2}$ is a milestone in solid state physics and materials science. It has opened the path for the synthesis of a new class of materials with novel physical properties. The most prominent member, grapheme-a monoatomic layer of crystalline $\mathrm{C}$ with hexagonal structure-is obtained by micromechanical cleavage of graphite as a three-dimensional (3D) layered parent material. Likewise the $2 \mathrm{D}$ hexagonal boron nitride (h-BN) is produced from 3D h-BN. Recently a single layer of h-BN has been fabricated by controlled energetic electron irradiationinduced layer by layer sputtering. ${ }^{3}$ While graphene is a metallic conductor, $2 \mathrm{D} \mathrm{h}-\mathrm{BN}$ is a true insulator ${ }^{1}$ (3D BN has a direct band gap in the ultraviolet region ${ }^{4}$ ). Graphene and 2D h-BN also differ from the point of view chemical bonding. Graphene is a purely covalent material, while BN, built from III-V elements in the periodic table, is a material with covalent and partially ionic bonding. Both graphene and 2D h-BN can be regarded as building material for one-dimensional (1D) nanotubes (for a review see Refs. 5-7). For many reasons, knowledge of the phonon spectrum is most useful. In case of graphene, the phonon spectrum has been determined from in-plane inelastic x-ray scattering (IXS) on single crystals of graphite. ${ }^{8}$ The experimental data allow to derive a force constant model for the covalent bonding of graphene. ${ }^{8}$ The force constant model in turn can be used to calculate by analytic methods elastic tension coefficients of graphene ${ }^{9}$ and to relate the latter to IXS scattering results. ${ }^{10}$ In case of h-BN, phonon spectra have been measured by highresolution electron energy loss spectroscopy (HREELS) (Ref. 11) of an epitaxial monolayer film of h-BN on Ni(111). Elastic constants ${ }^{12}$ and phonon dispersion relations ${ }^{13}$ of bulk h-BN have been determined by IXS and analyzed by $a b$ initio calculations. Theoretical work on $\mathrm{h}-\mathrm{BN}$ has been motivated by the fabrication of $\mathrm{BN}$ nanotubes. ${ }^{14,15}$ Due to the presence of Coulomb forces, the lattice dynamics of ionic crystals is more subtle than in the case of pure covalent bonding. ${ }^{16,17}$ Phonon dispersion relations of $3 \mathrm{D} \mathrm{h}-\mathrm{BN},{ }^{18-20}$ of h-BN monolayers ${ }^{21-23}$ and of nanotubes ${ }^{22,24}$ have been obtained by first principles and tight binding numerical calculations. In particular Ref. 22 comprises also extensive numerical results on the elastic properties of $2 \mathrm{D} \mathrm{h}-\mathrm{BN}$.

In the present theoretical work we rather will put emphasis on the analytic formulation of the lattice dynamics, including elastic and piezoelectric phenomena, within a rigidion model. In that respect our work is complementary to the previous, more computational based results on $2 \mathrm{D}$ h-BN. The restriction to a rigid-ion model at this stage is justified. Indeed so far we know of no experimental results on the lattice dynamics of free-standing 2D h-BN which would allow us to determine the parameters of a more sophisticated theoretical model. As we will show, a comparison with experiment has to be based on an extrapolation from results on $3 \mathrm{D}$ h-BN. On the other hand the relatively simple rigid-ion model allows us to treat in a rigorous way important qualitative aspects which follow from the two-dimensionality and the hexagonal structure of the crystal. The content of the paper is the following. First (Sec. II) we set up the dynamical matrix of the 2D ionic crystal as sum of a covalent forces part and an electrostatic Coulomb part. The covalent part is constructed with a force constant model related to the one used for graphene. ${ }^{8}$ The Coulomb part is based on the Ewald method applied to a $2 \mathrm{D}$ crystal, whereby a nonanalytic term is isolated. Phonon dispersions are calculated. In Sec. III Born's long-wavelength method is used to calculate the elastic constants (tension coefficients in two dimensions). Special emphasis is put on the role of internal strains. Also the elastic bending coefficient is calculated. A comparison of various theoretical and experimental results is made. Next (Sec. IV) we study piezoelectric phenomena in the $2 \mathrm{D}$ ionic hexagonal crystal. In Sec. $\mathrm{V}$ the dispersion of the optical phonon branches near the $\Gamma$ point of the Brillouin zone is studied by analytic means, the role of the nonanalytic Coulomb term 


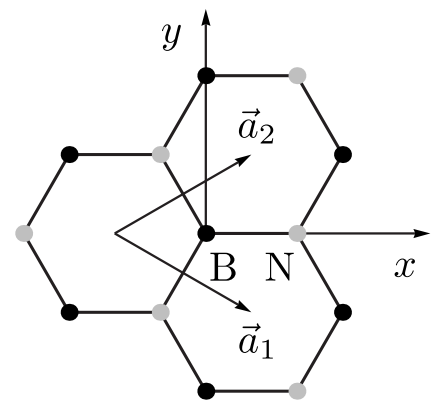

FIG. 1. Structural detail from a h-BN monolayer; $\vec{a}_{1}$ and $\vec{a}_{2}$ primitive lattice vectors.

and overbending is elucidated. Concluding remarks close the paper (Sec. VI).

\section{PHONONS IN 2D HEXAGONAL BN}

We recall some basic concepts of the lattice dynamics of ionic crystals, adapted to the case of two dimensions. Taking into account covalent and Coulomb interactions we calculate the phonon dispersion relations for 2D h-BN. We start from an infinite 2D hexagonal crystal in the $(x, y)$ plane (Fig. 1), with one $\mathrm{B}$ and one $\mathrm{N}$ atom per unit cell. The position of the $\vec{n}_{\perp}$-th unit cell is given by the lattice vector $\vec{X}\left(\vec{n}_{\perp}\right)=n_{1} \vec{a}_{1}+n_{2} \vec{a}_{2}, \vec{n}_{\perp}$ stands for the pair of integers $\left(n_{1}, n_{2}\right)$, $\vec{a}_{1}$ and $\vec{a}_{2}$ are primitive lattice vectors. The $2 \mathrm{D}$ Brillouin zone with symmetry points $\Gamma, K, M$ is shown in Fig. 2 . The basic theoretical quantity in lattice dynamics is the dynamical matrix $D\left(\vec{q}_{\perp}\right)$, here $\vec{q}_{\perp}=\left(q_{x}, q_{y}\right)$ is the $2 \mathrm{D}$ wave vector. Here and in the following we will use the index $\perp$ for in-plane directions (i.e., perpendicular to the $\vec{c}$ axis of the $3 \mathrm{D}$ crystal), and the index $\|$ for the direction parallel to $\vec{c}$, i.e. out of plane. Since we allow for ionic displacements in the directions $(x, y)$ in plane and $z$ out of plane, the dynamical matrix is of dimension $6 \times 6$ and hence we will get three acoustic and three optical phonon branches. As usual ${ }^{16,17}$ we write $D$ as a sum of covalent contribution $F$ and a Coulomb contribution C,

$$
D_{i j}^{\kappa \kappa^{\prime}}\left(\vec{q}_{\perp}\right)=F_{i j}^{\kappa \kappa^{\prime}}\left(\vec{q}_{\perp}\right)+C_{i j}^{\kappa \kappa^{\prime}}\left(\vec{q}_{\perp}\right) .
$$

Here $\kappa\left(\kappa^{\prime}\right)$ labels the ions $\mathrm{B}, \mathrm{N}$ while $i(j) \in\{1,2,3\}$ is a Cartesian index for the $(x, y, z)$ directions. Given the struc-

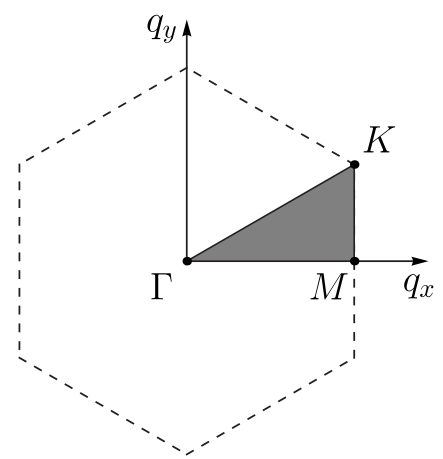

FIG. 2. 2D Brillouin zone. tural similarity between $2 \mathrm{D} \mathrm{h}-\mathrm{BN}$ and graphene and the locations of $\mathrm{B}, \mathrm{C}$, and $\mathrm{N}$ atoms in the periodic table, we evaluate the covalent dynamical matrix $F\left(\vec{q}_{\perp}\right)$ with an empirical force constant model which is related to a corresponding model for graphene. ${ }^{8}$ The dynamical matrix $C\left(\vec{q}_{\perp}\right)$ for Coulomb forces is calculated by means of Ewald's method (see, e.g., Refs. 16 and 17) which we have adapted to the case of a 2D ionic crystal. Thereby we have used a 3D Gaussian charge distribution with range parameter $\eta$ on each ion site. As a result we obtain

$$
C_{i j}^{\kappa \kappa^{\prime}}\left(\vec{q}_{\perp}\right)=\frac{1}{\sqrt{m_{\kappa} m_{\kappa^{\prime}}}} \Phi_{i j}^{\kappa \kappa^{\prime}}\left(\vec{q}_{\perp}\right)-\delta_{\kappa \kappa^{\prime}} \frac{1}{m_{\kappa}} \sum_{\kappa^{\prime \prime}} \Phi_{i j}^{\kappa \kappa^{\prime \prime}}\left(\vec{q}_{\perp}=\overrightarrow{0}\right),
$$

where

$$
\Phi_{i j}^{\kappa \kappa^{\prime}}\left(\vec{q}_{\perp}\right)=\left.\Phi_{i j}^{\kappa \kappa^{\prime}}\left(\vec{q}_{\perp}\right)\right|_{1}+\left.\Phi_{i j}^{\kappa \kappa^{\prime}}\left(\vec{q}_{\perp}\right)\right|_{2} .
$$

Here the first and the second term on the right-hand side (rhs) are given by summations over the reciprocal and direct 2D lattices, respectively. For in-plane displacements where $i, j \in\{x, y\}$ we have

$$
\begin{aligned}
\left.\Phi_{i j}^{\kappa \kappa^{\prime}}\left(\vec{q}_{\perp}\right)\right|_{1}= & \frac{2 \pi e_{\kappa}^{\perp} e_{\kappa^{\prime}}^{\perp}}{v_{2 \mathrm{D}}} \sum_{\vec{\tau}_{\perp}} \frac{\left(\vec{\tau}-\vec{q}_{\perp}\right)_{i}\left(\vec{\tau}-\vec{q}_{\perp}\right)_{j}}{\left|\vec{\tau}_{\perp}-\vec{q}_{\perp}\right|} e^{-i \vec{\tau}_{\perp} \cdot\left(\vec{r}^{\kappa}-\vec{r}^{\kappa^{\prime}}\right)} \\
& \times \operatorname{erfc}\left(\frac{\left|\vec{\tau}-\vec{q}_{\perp}\right|}{\sqrt{4 \eta}}\right), \\
\left.\Phi_{i j}^{\kappa \kappa^{\prime}}\left(\vec{q}_{\perp}\right)\right|_{2}= & -e_{\kappa}^{\perp} e_{\kappa^{\prime}}^{\perp} \eta^{3 / 2} \sum_{\vec{n}_{\perp}^{\prime}} H_{i j}^{\kappa \kappa^{\prime}}\left[\sqrt{\eta}\left|\vec{X}\left(\vec{n}_{\perp}^{\prime} \kappa^{\prime}\right)-\vec{X}\left(\vec{n}_{\perp} \kappa\right)\right|\right] \\
& \times e^{i \vec{q}_{\perp} \cdot\left[\vec{X}\left(\vec{n}_{\perp}^{\prime} \kappa^{\prime}\right)-\vec{X}_{\left.\left(\vec{n}_{\perp} \kappa\right)\right]}\right.}
\end{aligned}
$$

Here $m_{\kappa}$ is the mass of the $\kappa$ th ion, $e_{\kappa}^{\perp}$ its effective charge. The $2 \mathrm{D}$ vector $\vec{X}\left(\vec{n}_{\perp} \kappa\right)=\vec{X}\left(\vec{n}_{\perp}\right)+\vec{r}^{\kappa}$ denotes the equilibrium position of the ion in the 2D plane, where $\vec{X}\left(\vec{n}_{\perp}\right)$ is the lattice vector of the $\vec{n}_{\perp}$ th unit cell, $\vec{r}^{\kappa}$ the position vector of the $\kappa$ th ion in a unit cell. In Eq. (4) the summation runs over the 2D reciprocal lattice vectors $\vec{\tau}_{\perp}, v_{2 \mathrm{D}}=a^{2} \sqrt{3} / 2$ is the area of the 2D unit cell, where $a=a_{\mathrm{BN}} \sqrt{3}$ is the length of a primitive lattice vector, $a=\left|\vec{a}_{1}\right|=\left|\vec{a}_{2}\right|$, and $a_{\mathrm{BN}}$ the nearest neighbor distance. For 2D h-BN we take the bond length $a_{\mathrm{BN}}=1.446 \AA .{ }^{12}$ The function $H_{i j}^{\kappa \kappa^{\prime}}$ in Eq. (5) is defined by

$$
\begin{aligned}
H_{i j}^{\kappa \kappa^{\prime}}(\vec{x})= & \frac{x_{i} x_{j}}{x^{2}}\left[\frac{3}{x^{3}} \operatorname{erfc}(x)+\frac{2}{\sqrt{\pi}}\left(\frac{3}{x^{2}}+2\right) e^{-x^{2}}\right] \\
& -\delta_{i j}\left[\frac{1}{x^{3}} \operatorname{erfc}(x)+\frac{2}{\sqrt{\pi}} \frac{1}{x^{2}} e^{-x^{2}}\right] .
\end{aligned}
$$

For the case of out-of-plane displacements, $i=z, j=z$, one gets 

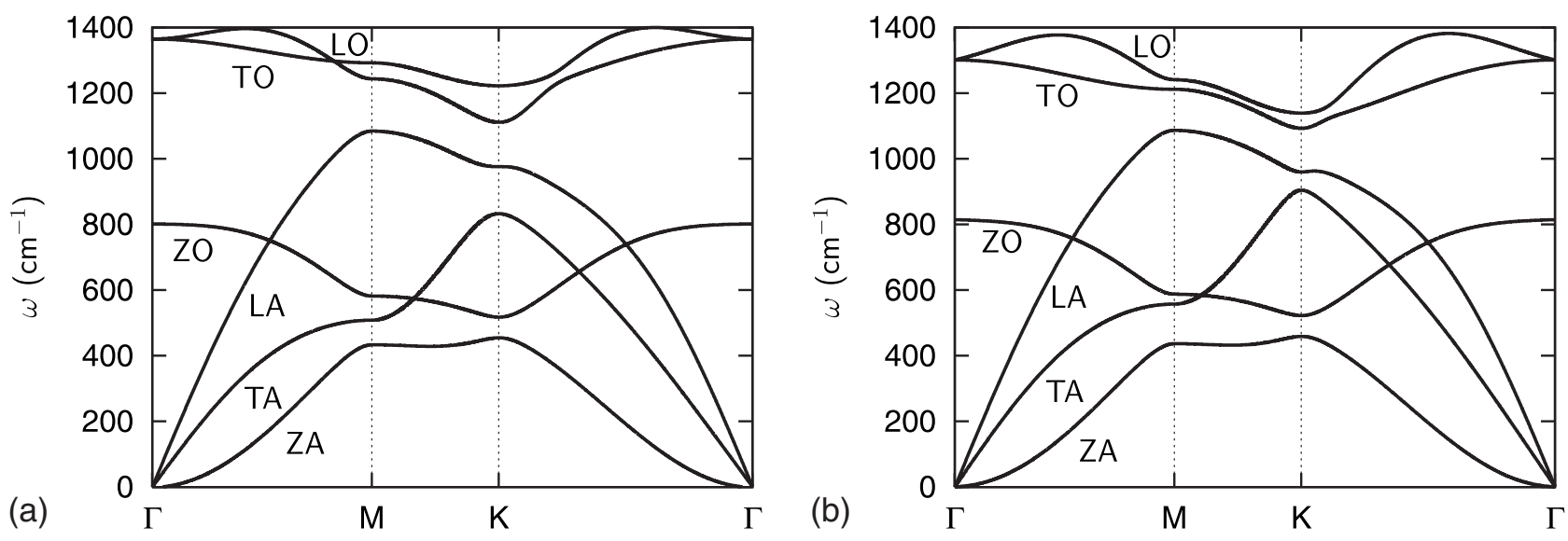

FIG. 3. Phonon dispersions of 2D h-BN, (a) without Coulomb forces, (b) Coulomb forces included.

$$
\begin{aligned}
\left.\Phi_{z z}^{\kappa \kappa^{\prime}}\left(\vec{q}_{\perp}\right)\right|_{1}= & \frac{2 \pi e_{\kappa}^{\|} e_{\kappa^{\prime}}^{\|}}{v_{2 \mathrm{D}}} \sum_{\vec{\tau}_{\perp}}\left[2 \sqrt{\frac{\eta}{\pi}} e^{-\left|\vec{\tau}_{\perp}-\vec{q}_{\perp}\right|^{2} / 4 \eta}-\mid \vec{\tau}_{\perp}\right. \\
& \left.-\vec{q}_{\perp} \mid \operatorname{erfc}\left(\frac{\vec{\tau}_{\perp}-\vec{q}_{\perp}}{\sqrt{4 \eta}}\right)\right] e^{i \vec{\tau}_{\perp} \cdot\left(\vec{r}^{\kappa^{\prime}}-\vec{r}^{\kappa}\right)} .
\end{aligned}
$$

The term $\left.\Phi_{z z}^{\kappa \kappa^{\prime}}\left(\vec{q}_{\perp}\right)\right|_{2}$ is still given by Eq. (5), however since the equilibrium positions of the ions are in a same plane, one has

$$
H_{z z}^{\kappa \kappa^{\prime}}(\vec{x})=-\left[\frac{1}{x^{3}} \operatorname{erfc}(x)+\frac{2}{\sqrt{\pi}} \frac{1}{x^{2}} e^{-x^{2}}\right],
$$

in addition the effective charges are $e_{\kappa}^{\|}$and $e_{\kappa^{\prime}}^{\|}$. The elements $C_{i z}^{\kappa \kappa^{\prime}}\left(\vec{q}_{\perp}\right), i \in x, y$, are zero.

We note, as is the case for $3 \mathrm{D}$ ionic crystals, that the divergent term arising in Eq. (5) when $\left(\vec{n}_{\perp}^{\prime} \kappa^{\prime}\right)=\left(\vec{n}_{\perp} \kappa\right)$ is compensated by the same term from $\Phi_{i j}^{\kappa \kappa}\left(\vec{q}_{\perp}=\overrightarrow{0}\right)$ in Eq. (2). On the other hand the 2D case is different as far as the term arising from $\vec{\tau}=\overrightarrow{0}$ in Eq. (4) is concerned in the longwavelength limit. The contributions to the dynamical matrix for in-plane motion are given by

$$
\begin{aligned}
C_{i j}^{\kappa \kappa^{\prime}}\left(\vec{q}_{\perp}, \vec{\tau}=\right. & \overrightarrow{0})\left.\right|_{1}=\frac{2 \pi e_{\kappa}^{\perp} e_{\kappa^{\prime}}^{\perp}}{v_{2 \mathrm{D}}\left(m_{\kappa^{\prime}} m_{\kappa^{\prime}}\right)^{1 / 2}} \frac{\left(\vec{q}_{\perp}\right)_{i}\left(\vec{q}_{\perp}\right)_{j}}{\left.\mid \vec{q}_{\perp}\right) \mid}\left(1-\frac{\left|\vec{q}_{\perp}\right|}{\sqrt{\pi \eta}}\right) \\
& +O\left(\left|\vec{q}_{\perp}\right|^{3}\right)
\end{aligned}
$$

$i(j) \in\{x, y\}$. The contribution for the out-of-plane motion in the long-wavelength limit is obtained from Eq. (7) and reads

$$
\begin{aligned}
C_{z z}^{\kappa \kappa^{\prime}}\left(\vec{q}_{\perp}, \vec{\tau}=\right. & \overrightarrow{0})\left.\right|_{1}=-\frac{2 \pi e_{\kappa}^{\|} e_{\kappa^{\prime}}^{\|}}{v_{2 \mathrm{D}}\left(m_{\kappa} m_{\kappa^{\prime}}\right)^{1 / 2}}\left|\vec{q}_{\perp}\right|\left(1-\frac{1}{2} \frac{\left|\vec{q}_{\perp}\right|}{\sqrt{\pi \eta}}\right) \\
& +O\left(\left|\vec{q}_{\perp}\right|^{3}\right) .
\end{aligned}
$$

Both expressions $\left.C_{i j}^{\kappa \kappa^{\prime}}\right|_{1}$ and $\left.C_{z z}^{\kappa \kappa^{\prime}}\right|_{1}$ have a unique limit 0 for $\vec{q}_{\perp} \rightarrow \overrightarrow{0}$, however they are not analytic since their derivatives with respect to the wave vector components are discontinuous at the $\Gamma$ point. The wave number dependence is a consequence of the two-dimensionality but does not depend otherwise on the symmetry of the crystal. A similar behavior is found for the dynamical matrix of the 2D Wigner crystal. ${ }^{25}$

The long-wavelength Coulomb part of the dynamical matrix in three-dimensional ionic crystals is obtained by performing Fourier transforms in 3D space. ${ }^{16,17}$ One finds that $C_{i j}^{\kappa \kappa^{\prime}}(\vec{q}, \vec{\tau}=\overrightarrow{0})$ is proportional to $\left(1 / v_{3 \mathrm{D}}\right) q_{i} q_{j} / q^{2}$, where $i(j) \in\{x, y, z\}, v_{3 \mathrm{D}}$ is the volume of the 3D unit cell, $q^{2}=q_{x}^{2}+q_{y}^{2}+q_{z}^{2}$. The limit value depends on the direction of $\vec{q} \rightarrow \overrightarrow{0}$. This feature translates the sample shape dependence of the Coulomb term in three dimensions, ${ }^{26}$ the crystal point group symmetry at $\Gamma$ is broken. The Coulomb term is usually treated as a symmetry-breaking macroscopic field ${ }^{16}$ and leads to a splitting of the highest longitudinal and transverse optical modes at the $\Gamma$ point in cubic ionic crystals. ${ }^{27}$ On the other hand, since the Coulomb term vanishes in $2 \mathrm{D}$, there is no symmetry breaking and the phonon frequencies at the $\Gamma$ point can be classified by group theory. Since the point group of $2 \mathrm{D}$ h-BN is $D_{3 h}$, the three optical phonons at $\Gamma$ belong to the irreducible representations $A_{2}^{\prime \prime}+E^{\prime}$. Here $A_{2}^{\prime \prime}$ corresponds to the out-of-plane vibrational $(\mathrm{ZO})$ mode while $E^{\prime}$ corresponds to the degenerate in-plane LO (longitudinal optical) and TO (transverse optical) modes. The degeneracy of LO and TO at $\Gamma$ is well confirmed by numerical calculations ${ }^{22}$ on 2D h-BN. The HREELS results of Rokuta et al. ${ }^{11}$ on an epitaxial monolayer film of h-BN on $\mathrm{Ni}(111)$ exhibit no splitting of LO and TO at $\Gamma$, in agreement with theory. In Sec. V we will show that the nonanalytic part of the Coulomb dynamical matrix in the 2D case leads to a linear $\vec{q}_{\perp}$ dependence with positive slope for the LO phonon dispersion, negative slope for the ZO (out-of-plane) optical phonon branch, while the TO phonon branch remains flat.

The phonon dispersion relations are obtained from the solution of the secular equation

$$
\left|1 \omega^{2}-D\left(\vec{q}_{\perp}\right)\right|=0 .
$$

Here 1 is the $6 \times 6$ unit matrix. In Fig. 3(a) we show the phonon branches obtained solely from the covalent forces dynamical matrix $F$. For the calculation of the matrix $F$ we refer to Appendix A, Ref. 9 where now we have to use the masses $m_{\mathrm{B}}=10.81$ and $m_{\mathrm{N}}=14.01$ (atomic mass units). The force parameters which mimic the covalent bond forces are $f_{r}^{(n)}, f_{i}^{(n)}$, and $f_{o}^{(n)}$, with $n=1, \ldots, 5$. They represent the radial 
TABLE I. Covalent force constant parameters, units $\mathrm{eV} / \AA^{2}$.

\begin{tabular}{rrrr}
\hline$n$ & \multicolumn{1}{c}{$f_{r}^{(n)}$} & \multicolumn{1}{c}{$f_{i}^{(n)}$} & \multicolumn{1}{c}{$f_{o}^{(n)}$} \\
\hline 1 & 21.998 & 5.010 & 5.255 \\
2 & 3.431 & -1.811 & -0.418 \\
3 & -2.564 & 2.349 & 0.439 \\
4 & 0.479 & 0.077 & -0.443 \\
5 & 0.880 & 0.099 & 0.094 \\
\hline \hline
\end{tabular}

(r) bond-stretching, in-plane (i) and out-of-plane (o) tangential bond-bending forces between the $n$th neighbors, respectively. ${ }^{5,8}$ In comparison with graphene, we have reduced the strength of all force constants $f_{r}^{(n)}$ and $f_{o}^{(n)}$ by $15 \%$ and of $f_{i}^{(n)}$ by $40 \%$ (see Table I). Such a reduction of the covalent forces is inferred from a comparison of the IXS results on phonon dispersions in graphite $^{8}$ and h-BN. ${ }^{13} \mathrm{Al}-$ though the two $2 \mathrm{D}$ crystals are isoelectronic and isostructural, the in-plane bonding in the homonuclear graphene is stronger than in the heteronuclear $2 \mathrm{D}$ h-BN. While the reduction of force constants leads to an overall scaling down of the phonon frequencies, the major qualitative changes between the dispersions shown in Fig. 3(a) and the corresponding results for graphene ${ }^{8,9}$ are due to the different masses. The results shown in Fig. 3(b) have been obtained with inclusion of the Coulomb part $C$ of the dynamical matrix. We have used effective Born charges $e_{B}^{\perp}=-e_{N}^{\perp}=-0.60 e$, $e_{B}^{\|}=-e_{N}^{\|}=-0.15 e$ ( $e$ is the elementary charge $)$, in both cases the static dielectric constant is taken $\varepsilon_{0}=1$. The corresponding quantities in Ref. 22 are $\left(Z_{\|}^{B} / \sqrt{\varepsilon_{0}}\right)=-1.35 e$ (in plane) and $\left(Z_{\perp}^{B} / \sqrt{\varepsilon_{0}}\right)=-0.41 e$ (out of plane), where in addition the electrostatic dipole-dipole interaction is considerably reduced by a switching function. Recent $a b$ initio calculations suggest a charge transfer $\Delta Q=0.429$ electrons from $\mathrm{B}$ to $\mathrm{N}$ atoms in $2 \mathrm{D}$ h-BN. ${ }^{28}$ Effective charges and dielectric constants have been calculated for $3 \mathrm{D}$ h-BN by a first-principles study. ${ }^{19}$ However there still remain quantitative discrepancies with experiments $^{29}$ on bulk h-BN, so far we know of no optical phonon results on $2 \mathrm{D} \mathrm{h}-\mathrm{BN}$.

Our choice of the valence force constants and of the effective charges leads to reasonable values of the optical phonon frequencies at the $\Gamma$ point. Indeed our calculated values for the frequencies of the degenerate LO and TO in-plane modes $\left(1301 \mathrm{~cm}^{-1}\right)$ and for the $\mathrm{ZO}$ out-of-plane mode $\left(814 \mathrm{~cm}^{-1}\right)$ at $\Gamma$ are close to the corresponding experimental values 1371 and $790 \mathrm{~cm}^{-1}$ of 2D h-BN on Ni(111) (Ref. 11) and to the $E_{2 g}$ Raman mode (1370 $\mathrm{cm}^{-1}$ ) (Refs. 29-31) and $A_{2 u}$ IR mode $\left(783 \mathrm{~cm}^{-1}\right)$ in $3 \mathrm{D} \mathrm{h}-\mathrm{BN} .{ }^{29}$ Comparing our results with the $a b$ initio calculated phonon spectra of $2 \mathrm{D}$ h-BN in Ref. 22 and with the in-plane IXS results on 3D h-BN, ${ }^{13}$ we note an overall satisfactory similarity for the evolution of the phonon branches ZA (acoustic out of plane), LA (longitudinal acoustic), TA (transverse acoustic) and ZO. As a quantitative difference we mention that our ZA branches are too high at the $K$ and $M$ points of the Brillouin zone. In agreement with Ref. 13, we obtain a crossing of the $\mathrm{ZO}$ and TA branches near $M$ on the $\overline{M K}$ axis while in Ref. 22 these three branches cross on the $\overline{\Gamma M}$ axis near $M$. Concerning the highest optical phonon branches $\mathrm{LO}$ and $\mathrm{ZO}$, our results agree with Ref. 22 on two important points; (i) the LO and TO modes are degenerate at $\Gamma$, as should be the case in two dimensions, (ii) comparing Figs. 3(a) and 3(b), we see that the Coulomb interaction increases the overbending of the LO phonon branch. We will return to these points in Sec. V. As a qualitative difference with Refs. 22 and 13, we find that the Coulomb interaction prevents a crossing of the LO and TO modes near $M$ or $K$. This discrepancy could be due to a shortcoming of our use of a rigid-ion model. In any case additional experimental information on the evolution of the LO and TO modes is needed. In the next Section we will study the elastic constants and compare our results with theory and experiment.

\section{ELASTIC CONSTANTS}

Having obtained analytical expressions of the dynamical matrix in the last section, we calculate the elastic constants by means of Born's method of long waves. ${ }^{16}$ One starts from a series expansion of the dynamical matrix in powers of small $\vec{q}_{\perp}$ :

$$
\begin{aligned}
D_{i j}^{\kappa \kappa^{\prime}}\left(\vec{q}_{\perp}\right)= & D_{i j}^{\kappa \kappa^{\prime}(0)}+i \sum_{k} D_{i j, k}^{\kappa \kappa^{\prime}(1)} q_{k}+\frac{1}{2} \sum_{k, l} D_{i j, k l}^{\kappa \kappa^{\prime}(2)} q_{k} q_{l} \\
& +\left.C_{i j}^{\kappa \kappa^{\prime}}\left(\vec{q}_{\perp}, \tau \overrightarrow{=} \overrightarrow{0}\right)\right|_{1},
\end{aligned}
$$

where $k, l \in\{x, y\}, \kappa, \kappa^{\prime} \in\{\mathrm{B}, \mathrm{N}\}$. Here

$$
\begin{aligned}
& D_{i j}^{\kappa \kappa^{\prime}(0)}=F_{i j}^{\kappa \kappa^{\prime}(0)}+C_{i j}^{\kappa \kappa^{\prime}(0)}, \\
& D_{i j}^{\kappa \kappa^{\prime}(1)}=F_{i j}^{\kappa \kappa^{\prime}(1)}+C_{i j}^{\kappa \kappa^{\prime}(1)}, \\
& D_{i j}^{\kappa \kappa^{\prime}(2)}=F_{i j}^{\kappa \kappa^{\prime}(2)}+C_{i j}^{\kappa \kappa^{\prime}(2)},
\end{aligned}
$$

where we have separated off explicitly the contribution $\left.C_{i j}^{\kappa \kappa^{\prime}}\left(\vec{q}_{\perp}, \vec{\tau}=\overrightarrow{0}\right)\right|_{1}$, Eqs. (9) and (10). Notice the presence of the $D^{(1)}$ term in Eq. (12). This term is due to the absence of a center of inversion in the $2 \mathrm{D}$ crystal. It accounts for the relative displacements of the $\mathrm{B}$ and $\mathrm{N}$ sublattices.

It is convenient to introduce three acoustic displacement vectors in six dimensions,

$$
w^{(j)}(\kappa i)=\delta_{i j} \sqrt{\frac{m_{\kappa}}{m}},
$$

where $i(j)=1,2,3, \kappa=\mathrm{B}, \mathrm{N}$, and $m=m_{\mathrm{B}}+m_{\mathrm{N}}$ is the mass per unit cell. The corresponding three optical displacement vectors read

$$
\xi^{(\lambda)}(\kappa i)=\delta_{\lambda i} \operatorname{sgn} \kappa \sqrt{\frac{\mu}{m_{\kappa}}},
$$

$\lambda=1,2,3$, where $\operatorname{sgn} \kappa=+1$ for $\kappa=\mathrm{B}$ and -1 for $\kappa=\mathrm{N}$, $\mu=m_{\mathrm{B}} m_{\mathrm{N}} / m$ is the reduced mass. The matrix $D^{(0)}$ is symmetric with

$$
D_{i j}^{\kappa \kappa^{\prime}(0)}=\delta_{i j} D_{i i}^{\kappa \kappa^{\prime}(0)},
$$

and $D^{\mathrm{BN}(0)}=D^{\mathrm{NB}(0)}=-\sqrt{m_{\mathrm{B}} / m_{\mathrm{N}}} D^{\mathrm{BB}(0)}$. The optical phonon frequencies at the $\Gamma$ point are given by $\vec{\xi}^{(\lambda) t} \cdot D^{(0)} \cdot \vec{\xi}^{\left(\lambda^{\prime}\right)}$ 
$\equiv \delta_{\lambda \lambda^{\prime}} \hat{D}_{\lambda \lambda}^{(0)}$. The superscript ${ }^{t}$ stands for transposed. Explicitly one has

$$
\hat{D}_{\lambda \lambda^{\prime}}^{(0)} \equiv \sum_{i} \sum_{\kappa \kappa^{\prime}} \xi^{\left(\lambda^{\prime}\right)}(\kappa i) D_{i i}^{\kappa \kappa^{\prime}(0)} \xi^{(\lambda)}\left(\kappa^{\prime} i\right)=\delta_{\lambda \lambda^{\prime}}\left(\omega_{\lambda}^{(0)}\right)^{2},
$$

where $\omega_{1}^{(0)}=\omega_{2}^{(0)}$, with $\omega_{1}^{(0)} \equiv \omega_{\mathrm{LO}}^{(0)}, \quad \omega_{2}^{(0)} \equiv \omega_{\mathrm{TO}}^{(0)}, \quad$ are $\quad$ the degenerate in-plane optical modes at $\Gamma$ and where $\omega_{3}^{(0)} \equiv \omega_{\mathrm{ZO}}^{(0)}<\omega_{1}^{(0)}$ is the out-of-plane optical mode. The elements $D_{i j, k}^{\kappa \kappa^{\prime}(1)}, k \in\{1,2\}$ fixed, are different from zero only for noncentrosymmetric crystals such as graphene and $2 \mathrm{D}$ h-BN. Only elements with $\kappa \neq \kappa^{\prime}$, i.e. corresponding to different sublattices are nonzero. Hermiticity of the dynamical matrix implies

$$
D_{i j, k}^{\mathrm{BN}(1)}=-D_{j i, k}^{\mathrm{NB}(1)} .
$$

Transforming the matrix $D_{\ldots, k}^{(1)}$ by means of the optical or (and) acoustic displacement vectors, we obtain $\hat{D}_{\lambda \lambda^{\prime}, k}^{(1)}$ $\equiv \vec{\xi}^{(\lambda) t} \cdot D_{. ., k}^{(1)} \cdot \vec{\xi}^{\left(\lambda^{\prime}\right)}=0, \quad \hat{D}_{i j, k}^{(1)} \equiv \vec{w}^{(i) t} \cdot D_{. ., k}^{(1)} \cdot \vec{w}^{(j)}=0 . \quad$ Only the mixed term $\hat{D}_{\lambda j, k}^{(1)} \equiv \vec{\xi}^{(\lambda) t} \cdot D_{\ldots, k}^{(1)} \cdot \vec{w}^{(j)}$, i.e.,

$$
\hat{D}_{\lambda j, k}^{(1)} \equiv \sum_{\kappa \kappa^{\prime}} \sum_{i i^{\prime}} \xi^{(\lambda)}(\kappa i) D_{i i^{\prime}, k}^{\kappa \kappa^{\prime}(1)} w^{(j)}\left(\kappa^{\prime} i^{\prime}\right)=D_{\lambda j, k}^{\mathrm{BN}(1)},
$$

where $\lambda \in\{1,2\}, j \in\{1,2\}$, is nonzero, with $\hat{D}_{\lambda j, k}^{(1)}=-\hat{D}_{j \lambda, k}^{(1)}$. The elements $D_{\lambda j, k}^{\mathrm{BN}(1)}$ lead to a coupling between optical and acoustic displacements in the long-wavelength regime.

Deformations of the crystal due to relative displacements of the sublattices, in casu $\mathrm{N}$ and $\mathrm{B}$, are known as internal strains. ${ }^{16}$ While it is evident to relate the second-order terms $D_{i j, k l}^{\kappa \kappa^{\prime}(2)}$ in the expansion Eq. (12) to the elastic constants, the perturbation method for the calculation of the elastic constants $C_{i j, k l}$ where external and internal strains are taken into account, is originally due to Born. One has ${ }^{16}$

$$
C_{i j, k l}=[i k, j l]+[j k, i l]-[i j, k l]+(i j, k l) .
$$

Thereby one defines "square brackets" and "round brackets" quantities, which in the $2 \mathrm{D}$ case read

$$
[i j, k l]=\frac{1}{v_{2 \mathrm{D}}} \sum_{\kappa \kappa^{\prime}}\left(m_{\kappa} m_{\kappa^{\prime}}\right)^{(1 / 2)} D_{i j, k l}^{\kappa \kappa^{\prime}(2)},
$$

and

$$
\begin{aligned}
(i j, k l)= & -\frac{1}{v_{2 \mathrm{D}}} \sum_{\kappa \kappa^{\prime}} \sum_{h p} \Gamma_{h p}^{\kappa \kappa^{\prime}}\left[\sum_{\kappa^{\prime \prime}}\left(m_{\kappa^{\prime \prime}}\right)^{1 / 2} D_{h i, k}^{\kappa \kappa^{\prime \prime}(1)}\right] \\
& \times\left[\sum_{\kappa^{\prime \prime \prime}}\left(m_{\kappa^{\prime \prime \prime}}\right)^{1 / 2} D_{p j, l}^{\kappa^{\prime} j^{\prime \prime \prime}(1)}\right],
\end{aligned}
$$

with

$$
\Gamma_{h p}^{\kappa \kappa^{\prime}}=\sum_{\lambda} \frac{\xi^{(\lambda)}(\kappa h) \xi^{(\lambda)}\left(\kappa^{\prime} p\right)}{\left(\omega_{\lambda}^{(0)}\right)^{2}} .
$$

These expressions become particularly transparent in terms of the acoustic and optical eigenvectors representation:

$$
[i j, k l]=\frac{\rho_{2 \mathrm{D}}}{2} \hat{D}_{i j, k l}^{(2)},
$$

where $\hat{D}_{i j, k l}^{(2)}=\vec{w}^{(i) t} \cdot D_{. ., k l}^{(2)} \cdot \vec{w}^{(j)}$; similarly

$$
(i k, j l)=-\rho_{2 \mathrm{D}} \sum_{\lambda} \hat{D}_{\lambda i, k}^{(1)} \frac{1}{\left(\omega_{\lambda}^{(0)}\right)^{2}} \hat{D}_{\lambda j, l}^{(1)} .
$$

Here $\rho_{2 \mathrm{D}}=m / v_{2 \mathrm{D}}$ is the (surface-) mass density.

There are two independent elastic constants of a 2D hexagonal crystal, they have the dimension of a surface tension coefficient. Using Voigt's notation, we write $\gamma_{11}$ for $C_{11,11}$ and $\gamma_{12}$ for $C_{11,22}$;

$$
\begin{gathered}
\gamma_{11}=[11,11]_{2 \mathrm{D}}+(11,11)_{2 \mathrm{D}}, \\
\gamma_{12}=2[12,12]_{2 \mathrm{D}}-[11,22]_{2 \mathrm{D}}+(11,22)_{2 \mathrm{D}} .
\end{gathered}
$$

Here the square brackets stand for

$$
[11, i i]_{2 \mathrm{D}}=\frac{1}{2 v_{2 \mathrm{D}}}\left[m_{B} D_{11, i i}^{\mathrm{BB}(2)}+m_{N} D_{11, i i}^{\mathrm{NN}(2)}+2\left(m_{B} m_{N}\right)^{1 / 2} D_{11, i i}^{\mathrm{NB}(2)}\right],
$$

with $i=1$ or 2 . Symmetry of the crystal structure implies

$$
[11,11]_{2 \mathrm{D}}-[11,22]_{2 \mathrm{D}}=2[12,12]_{2 \mathrm{D}} \text {. }
$$

The round brackets are given by

$$
\begin{aligned}
& (11,11)_{2 \mathrm{D}}=-\rho_{2 \mathrm{D}} \frac{1}{\left(\omega_{1}^{(0)}\right)^{2}}\left(D_{11,1}^{\mathrm{BN}(1)}\right)^{2}, \\
& (12,12)_{2 \mathrm{D}}=-\rho_{2 \mathrm{D}} \frac{1}{\left(\omega_{2}^{(0)}\right)^{2}}\left(D_{21,2}^{\mathrm{BN}(1)}\right)^{2},
\end{aligned}
$$

with $(11,11)_{2 \mathrm{D}}=(12,12)_{2 \mathrm{D}}=-(11,22)_{2 \mathrm{D}}$. These symmetry relations imply that the elastic constant $C_{12,12}$, i.e.,

$$
\gamma_{66}=[11,22]_{2 \mathrm{D}}+(12,12)_{2 \mathrm{D}},
$$

is obtained from the relation $\gamma_{11}-\gamma_{12}=2 \gamma_{66}$.

The longitudinal and transverse sound velocities for inplane displacements are related to the elastic tension coefficients by $c_{l}=\sqrt{\gamma_{11} / \rho_{2 \mathrm{D}}}$ and $c_{t}=\sqrt{\gamma_{66} / \rho_{2 \mathrm{D}}}$, respectively.

We recall that $\gamma_{66} \equiv \mu_{2 \mathrm{D}}$ and $\gamma_{12} \equiv \lambda_{2 \mathrm{D}}$ play the role of shear modulus and Lamé coefficient, while $\gamma_{11}-\gamma_{66} \equiv B_{2 \mathrm{D}}$ is the bulk modulus. Since the elastic properties of a $2 \mathrm{D}$ hexagonal crystal are isotropic in the crystal plane, Young's modulus $Y_{2 \mathrm{D}}$ and Poisson's ratio $\sigma_{2 \mathrm{D}}$ read $^{32}$

$$
\begin{gathered}
Y_{2 \mathrm{D}}=\frac{4 B_{2 \mathrm{D}} \mu_{2 \mathrm{D}}}{B_{2 \mathrm{D}}+\mu_{2 \mathrm{D}}}, \\
\sigma_{2 \mathrm{D}}=\frac{B_{2 \mathrm{D}}-\mu_{2 \mathrm{D}}}{B_{2 \mathrm{D}}+\mu_{2 \mathrm{D}}} .
\end{gathered}
$$

We have carried out numerical calculations of the elastic properties. We use the same parameters as for the calculation of the phonon branches in the last section. Our results, together with extrapolations from 3D experimental data and results from $a b$ initio calculations, are quoted in Table II. 
TABLE II. Elastic tension coefficients, Young's modulus, bulk modulus (units $10^{4} \mathrm{dyn} / \mathrm{cm}$ ), sound velocities (units $\mathrm{km} / \mathrm{s}$ ) and Poisson ratio of $2 \mathrm{D}$ h-BN.

\begin{tabular}{|c|c|c|c|c|c|c|c|c|}
\hline & $\gamma_{11}$ & $\gamma_{12}$ & $\gamma_{66}$ & $Y_{2 \mathrm{D}}$ & $B_{2 \mathrm{D}}$ & $c_{l}$ & $c_{t}$ & $\sigma_{2 \mathrm{D}}$ \\
\hline Present theory & 33.47 & 8.92 & 12.28 & 31.09 & 21.20 & 21.00 & 12.71 & 0.267 \\
\hline \multirow[t]{2}{*}{ Results of Ref. 22} & $34.30^{\mathrm{a}}$ & $11.90^{\mathrm{a}}$ & $10.70^{\mathrm{a}}$ & $30.20^{\mathrm{a}}$ & $23.60^{\mathrm{a}}$ & 21.00 & 13.00 & $0.347^{\mathrm{a}}$ \\
\hline & $32.20^{\mathrm{b}}$ & $7.60^{\mathrm{b}}$ & $12.30^{\mathrm{b}}$ & $30.40^{\mathrm{b}}$ & $19.90^{\mathrm{b}}$ & & & $0.236^{\mathrm{b}}$ \\
\hline Estimations from 3D h-BN (Ref. 12) & 27.00 & 5.62 & 10.69 & 25.83 & 16.31 & 18.86 & 11.86 & 0.208 \\
\hline idem from $a b$ initio $^{19,20}$ & 31.71 & 5.64 & 13.04 & 30.71 & 18.67 & & & 0.178 \\
\hline
\end{tabular}

airect calculations.
${ }^{\mathrm{b}}$ Obtained from $c_{l}$ and $c_{t}$.

Note that the values of $\gamma_{11}$ and $\gamma_{66}$ in graphene are larger by roughly $20 \%$. $^{9}$

To our knowledge, there are no experimental data on the elastic properties of 2D h-BN, however the elastic moduli of 3D h-BN have been measured by IXS on single crystals. ${ }^{12}$ The elastic constants $c_{11}$ and $c_{66}$ (and hence $c_{12}=c_{11}-2 c_{66}$ ) are obtained from in-plane phonon dispersion measurements by determination of the sound velocities. From Raman scattering experiments under varying pressure on 3D h-BN (Ref. 30) we conclude that the in-plane interactions between ions (valence and electrostatic) are much stronger than the interactions between ions belonging to different planes. We then admit as a reasonable approximation (see also Ref. 8) that the sound velocities $\mathrm{V}(\mathrm{LA}[100])$ and $\mathrm{V}\left(\mathrm{TA}[100]_{\langle 1-20\rangle}\right)$ obtained from $3 \mathrm{D} h-\mathrm{BN}$ are representative for $2 \mathrm{D} \mathrm{h}-\mathrm{BN}$ too. We then have ${ }^{9}$

$$
\begin{aligned}
& \gamma_{11} \approx \frac{c}{2} c_{11}, \\
& \gamma_{66} \approx \frac{c}{2} c_{66},
\end{aligned}
$$

and similarly for $c_{12}$, where $c_{11}, c_{12}$, and $c_{66}$ are the 3D elastic constants and where $c / 2$ is the distance between next neighbor planes in the $3 \mathrm{D}$ system. Using the values $c$ $=6.660 \AA$ and $c_{11}=811 \mathrm{GPa}, c_{66}=321 \mathrm{GPa}$ from Ref. 12, we arrive at the estimations of $\gamma_{11}, \gamma_{12}$, and $\gamma_{66}$ quoted in Table II. In a similar way we proceed for the elastic constants from $a b$ initio calculations on 3D h-BN. ${ }^{19,20}$ Given the values of $\gamma_{11}, \gamma_{12}$, and $\gamma_{66}$, the expressions for $B_{2 \mathrm{D}}, Y_{2 \mathrm{D}}$, and $\sigma_{2 \mathrm{D}}$ are calculated by means of the above formulas for the $2 \mathrm{D}$ hexagonal crystal. In comparing the relative contributions of the square brackets and round brackets terms (internal strains) with the elastic tension coefficients, we find that the latter account for a reduction of the values of $\gamma_{11}$ and $\gamma_{66}$ by $\approx 12 \%$ and $\approx 30 \%$, respectively. We also find that the contributions of the Coulomb interactions are rather small $(\approx 3 \%)$ in comparison with the valence forces contributions.

In order to calculate the bending coefficient $\kappa_{b}$ we proceed as in the theory of bending of thin plates $^{33}$ and membranes. ${ }^{32}$ Expanding the out-of-plane displacement components of the dynamical matrix up to fourth order in the wave vector components, we get

$$
D_{33}^{\kappa \kappa^{\prime}}\left(\vec{q}_{\perp}\right)=\frac{1}{4 !} D_{33,1111}^{\kappa \kappa^{\prime}(4)}\left(q_{x}^{2}+q_{y}^{2}\right)^{2}
$$

We transform again to the acoustic basis obtaining

$$
\hat{D}_{33}^{(4)}=\sum_{\kappa \kappa^{\prime}} w^{(3)}(\kappa 3) D_{33,1111}^{\kappa \kappa^{\prime}(4)} w^{(3)}\left(\kappa^{\prime} 3\right) .
$$

The bending coefficient is then given by

$$
\kappa_{b}=\frac{1}{4 !} \rho_{2 \mathrm{D}} \hat{D}_{33}^{(4)} \text {. }
$$

With the numerical input values given before, we obtain $\kappa_{b}=2.84 \times 10^{-12} \mathrm{erg}$ which is essentially due to valence forces; Coulomb interactions are found to be negligible. We note that the bending coefficient $\tilde{\kappa}_{b}$ for graphene in Ref. 9 is wrong by a factor 2, its value should read $\approx 3.6 \times 10^{-12} \mathrm{erg}$.

\section{PIEZOELECTRIC AND DIELECTRIC EFFECTS}

Since 2D h-BN is an ionic crystal where the lattice points are not centers of symmetry, the mechanical and the electrical properties are coupled. ${ }^{16,17} \mathrm{~A}$ central role is again played by the first-order expansion coefficients of the dynamical matrix, $D_{i j, k}^{\kappa \kappa^{\prime}(1)}$, Eq. (12). These coefficients lead to a coupling between acoustic and optical phonons in the longwavelength regime [see Eq. (21)]. The piezoelectric stress constants for the 2D case are obtained by means of Born's long-wavelength theory as

$$
e_{i, j l} \equiv[i, j l]=\frac{1}{v_{2 \mathrm{D}}} \sum_{\kappa \kappa^{\prime} \kappa^{\prime \prime}} \sum_{h} \sqrt{m_{\kappa}} D_{j h, l}^{\kappa \kappa^{\prime}(1)} \Gamma_{h i}^{\kappa^{\prime} \kappa^{\prime \prime}} \frac{e_{\kappa^{\prime \prime}}}{\sqrt{m_{\kappa^{\prime \prime}}}},
$$

with dimension charge/length and symmetry $e_{i, j l}=e_{i, l j}$. There exists only one independent piezoelectric stress constant in the case of $2 \mathrm{D}$ h-BN,

$$
e_{1,11}=\rho_{2 \mathrm{D}} D_{11,1}^{\mathrm{NB}(1)} \frac{1}{\left(\omega_{\mathrm{TO}}^{(0)}\right)^{2}} \frac{e_{B}}{\sqrt{m_{B} m_{N}}} .
$$

By structural symmetry it follows that

$$
e_{1,22}=e_{2,12}=-e_{1,11} .
$$

Numerical calculation with the model parameters of Sec. II give $e_{1,11}=-1.19 \times 10^{-12} \mathrm{C} / \mathrm{cm}$. Recent $a b$ initio numeri- 
cal work $^{34}$ on a flexoelectric effect in $2 \mathrm{D} \mathrm{h}$-BN quotes $e_{1,11}=-0.118 e / a_{B}=-3.57 \times 10^{-12}$ which is of the same order of magnitude. In order to compare our result with values of piezoelectric constants in 3D we use again dimensional arguments [see Eqs. (37) and (38)]. We consider the quantity

$$
\left.\frac{2}{c} e_{1,11} \equiv e_{1,11}\right|_{3 \mathrm{D}}
$$

where $c / 2=3.33 \AA$ is the distance between two neighboring planes in $3 \mathrm{D}$ h-BN. We obtain $\left.e_{1,11}\right|_{3 \mathrm{D}}=-0.357 \times 10^{-4} \mathrm{C} / \mathrm{cm}^{2}$ which is to be compared with the experimental value ${ }^{35}$ of the piezoelectric stress constant in $\alpha$ quartz, $e_{1,11}=0.171 \times 10^{-4} \mathrm{C} / \mathrm{cm}^{2}$.

The dielectric susceptibility for a $2 \mathrm{D}$ crystal in the longwavelength limit reads

$$
\chi_{i j}=\frac{1}{v_{2 \mathrm{D}}} \sum_{\kappa \kappa^{\prime}} \frac{e_{\kappa}}{\sqrt{m_{\kappa}}} \Gamma_{i j}^{\kappa \kappa^{\prime}} \frac{e_{\kappa^{\prime}}}{\sqrt{m_{\kappa^{\prime}}}} .
$$

In the present case only diagonal elements are nonzero,

$$
\chi_{i i}=\frac{\rho_{2 \mathrm{D}}}{\sqrt{m_{B} m_{N}}} \frac{\left(e_{B}^{(i)}\right)^{2}}{\left(\omega_{i}^{(0)}\right)^{2}},
$$

where $e_{B}^{\perp}=0.60 e$ for $i=1$ or $i=2$ with $\omega_{1}=\omega_{2}=\omega_{\mathrm{TO}}^{(0)}$ $=1301 \mathrm{~cm}^{-1}$ and $e_{\mathrm{B}}^{\|}=0.20 e$ for $i=3$ with $\omega_{3}=\omega_{\mathrm{ZO}}^{(0)}$ $=814 \mathrm{~cm}^{-1}$. We obtain the numerical values $\chi_{11}=\chi_{22}=5.51 \times 10^{-10} \mathrm{~cm}, \chi_{33}=0.40 \times 10^{-10} \mathrm{~cm}$.

In $3 \mathrm{D}$ crystals it is known that the piezoelectric coupling leads to a modification of the sound velocity. Here we have studied the coupled system of equations for acoustic and optical phonons, using all terms on the right-hand side of Eq. (12). Carrying out perturbation theory as shown in Ref. 36, Appendix B, we obtain a closed equation of motion for the acoustic displacements,

$$
\begin{aligned}
& \left\{\rho_{2 \mathrm{D}} \omega^{2} \delta_{i j}-q_{k} q_{l}\left[\gamma_{i k, j l}+2 \pi \frac{q_{s} q_{t}}{\left|\vec{q}_{\perp}\right|} e_{s, i l} e_{t, j k}\right]\right\} s_{j}\left(\vec{q}_{\perp}, \omega\right) \\
& =-\frac{F_{i}\left(\vec{q}_{\perp}, \omega\right)}{v_{2 \mathrm{D}}} .
\end{aligned}
$$

Here $s_{j}\left(\vec{q}_{\perp}, \omega\right)$ is the Fourier transform of the $j$ th component of the acoustic in-plane displacement, $F_{i}\left(\vec{q}_{\perp}\right)$ the $i$ th external mechanical force component. Explicitly we have

$$
\begin{aligned}
& \left\{\rho_{2 \mathrm{D}} \omega^{2}-q_{1}^{2}\left[\gamma_{11}+2 \pi \frac{q_{1}^{2}}{\left|\vec{q}_{\perp}\right|}\left(e_{1,11}\right)^{2}\right]\right\} s_{1}\left(\vec{q}_{\perp}, \omega\right) \\
& =-\frac{F_{1}\left(\vec{q}_{\perp}, \omega\right)}{v_{2 \mathrm{D}}}, \\
& \left\{\rho_{2 \mathrm{D}} \omega^{2}-q_{1}^{2}\left[\gamma_{66}+2 \pi \frac{q_{2}^{2}}{\left|\vec{q}_{\perp}\right|}\left(e_{2,12}\right)^{2}\right]\right\} s_{2}\left(\vec{q}_{\perp}, \omega\right) \\
& =-\frac{F_{2}\left(\vec{q}_{\perp}, \omega\right)}{v_{2 \mathrm{D}}} .
\end{aligned}
$$

Hence in 2D h-BN the corrections to the tension coefficients $\gamma_{11}$ and $\gamma_{66}$ and equivalently to the sound velocities vanish with $\vec{q}_{\perp}$ in the long-wavelength limit, while in the $3 \mathrm{D}$ crystal these corrections remain finite and depend in a complicated way on the direction of the wave. ${ }^{37}$ This difference between the $2 \mathrm{D}$ and the $3 \mathrm{D}$ crystal is a direct consequence of the different non-analytic behavior of the Coulomb part of the dynamical matrix as mentioned in Sec. II, Eq. (9). As a consequence of the vanishing of the piezoelectric corrections to $\gamma_{11}$ and $\gamma_{66}$, the elastic sum rule derived in Ref. 38 for the case of nonionic crystals is still valid and reads for $2 \mathrm{D} \mathrm{h}-\mathrm{BN}$

$$
\frac{v_{2 \mathrm{D}}}{m} \gamma_{i j, k l} \lim _{\vec{q}_{\perp} \rightarrow 0}\left[q_{j} q_{l} \int_{-\infty}^{+\infty} \frac{d \omega}{2 \pi} \frac{\hat{\chi}_{k p}\left(\vec{q}_{\perp}, \omega\right)}{\omega}\right]=\delta_{i p},
$$

where $\gamma_{11,11} \equiv \gamma_{11}$ and $\gamma_{12,12} \equiv \gamma_{66}$. The quantity $\hat{\chi}_{k p}\left(\vec{q}_{\perp}, \omega\right)$ is the acoustic dynamic displacement-displacement susceptibility.

\section{OVERBENDING OF OPTICAL PHONONS}

In graphene (in-plane phonon dispersions measured by inelastic $\mathrm{x}$-ray scattering on graphite ${ }^{8,39}$ ) it is found that the highest phonon branch which corresponds to a longitudinal optical phonon has maximum frequency away from the $\Gamma$ point. This phenomenon is known as overbending. ${ }^{7,40}$ Within the valence force model we find for graphene that overbending, characterized there by an upward curvature (i.e., a quadratic wave number dependence) when going from the $\Gamma$ point into the Brillouin zone, occurs only if fifth-order neighbors for in-plane stretching and bending forces are included. Nonetheless the highest optical phonon branches near the $\Gamma$ and the $K$ points of the Brillouin zone are still too flat in comparison with experimental results which show steeper slopes. ${ }^{8,39}$ The softening of the $E_{2 g}$ mode at $\Gamma$ (and of $A_{1}^{\prime}$ at $K)$ is attributed to strong electron-phonon coupling in $s p^{2}$ bonded carbon. ${ }^{39,41,42}$ These features have been identified and analyzed as Kohn anomalies. ${ }^{43}$ From experiment and $a b$ initio calculations it is inferred that near $\Gamma$ the frequency of the highest optical branch in graphene and graphite is of the form $\hbar \omega_{\vec{q}}=\hbar \omega_{\Gamma}+\alpha_{\Gamma} q+O\left(q^{2}\right)$. Equivalently the derivative of the frequency with respect to the wave vector exhibits a discontinuity at $\Gamma$, characterized by the coefficient $\alpha_{\Gamma}$ which is proportional to the square of the electron-phonon coupling. ${ }^{43}$

Since h-BN is an insulator, electron-phonon coupling and a concomittant Kohn anomaly should not cause a nonanalytic behavior of the dynamical matrix. However, as we have shown in Sec. II, the Coulomb interaction yields a nonanalytic contribution to the dynamical matrix in case of a $2 \mathrm{D}$ ionic crystal. Here we will show by a rigorous analytic calculation that the frequency of the LO phonon is of the form $\omega\left(\vec{q}_{\perp}\right)=\omega_{\Gamma}+\alpha\left|\vec{q}_{\perp}\right|+O\left(q_{\perp}^{2}\right)$. In Sec. III we have projected the dynamical matrix at $\Gamma$ onto the subspace of optical displacements, with the result

$$
\hat{D}_{\lambda \lambda^{\prime}}^{(0)}=\delta_{\lambda \lambda^{\prime}}\left(\omega_{\lambda}^{(0)}\right)^{2},
$$

where $\lambda\left(\lambda^{\prime}\right) \in\{1,2,3\}$. We recall that the in-plane LO and TO phonons are degenerate at $\Gamma: \omega_{\mathrm{LO}}^{(0)}=\omega_{\mathrm{TO}}^{(0)}$, while the outof-plane displacements have frequency $\omega_{\mathrm{ZO}}^{(0)}<\omega_{\mathrm{TO}}^{(0)}$. Similarly we project the matrix $\left[\left.C_{i j}^{\kappa \kappa^{\prime}}\left(\vec{q}_{\perp}, \vec{\tau}=\overrightarrow{0}\right)\right|_{1}\right]$ with elements given by Eqs. (9) and (10) onto the space of optical displacements, 


$$
\hat{C}_{\lambda \lambda^{\prime}}=\left.\vec{\xi}^{(\lambda) t} \cdot C\left(\vec{q}_{\perp}, \vec{\tau}=\overrightarrow{0}\right)\right|_{1} \cdot \vec{\xi}^{\left(\lambda^{\prime}\right)} .
$$

Restricting ourselves to terms of first and second order in the wave vector we obtain for the in-plane optical modes,

$$
\hat{C}_{i j}=\frac{2 \pi\left(e_{B}^{\perp}\right)^{2}}{v_{2 \mathrm{D}} \mu} \frac{q_{i} q_{j}}{\mid \vec{q}_{\perp}}\left(1-\frac{\left|\vec{q}_{\perp}\right|}{\sqrt{\pi \eta}}\right),
$$

for $i(j) \in\{1,2\}$ and for the out-of-plane optical mode

$$
\hat{C}_{z z}=-\frac{2 \pi\left(e_{B}^{\|}\right)^{2}}{v_{2 \mathrm{D}} \mu}\left|\vec{q}_{\perp}\right|\left(1-\frac{1}{2} \frac{\left|\vec{q}_{\perp}\right|}{\sqrt{\pi \eta}}\right) .
$$

Treating $\hat{C}$ as a perturbation to the optical dynamical matrix $\hat{D}^{(0)}$ at $\Gamma$, we solve the $3 \times 3$ secular determinant

$$
\left|\hat{D}_{\lambda \lambda^{\prime}}^{(0)}+\hat{C}_{\lambda \lambda^{\prime}}-\delta_{\lambda \lambda^{\prime}} \omega^{2}\right|=0
$$

We obtain the roots $\omega_{1}^{2}, \omega_{2}^{2}$, and $\omega_{3}^{2}$. The corresponding square roots are the perturbed optical phonon frequencies,

$$
\begin{gathered}
\omega_{\mathrm{LO}}\left(\vec{q}_{\perp}\right)=\omega_{\mathrm{LO}}^{(0)}+c_{\mathrm{LO}}\left|\vec{q}_{\perp}\right|\left(1-\frac{\left|\vec{q}_{\perp}\right|}{\sqrt{\pi \eta}}\right), \\
\omega_{\mathrm{TO}}\left(\vec{q}_{\perp}\right)=\omega_{\mathrm{TO}}^{(0)}, \\
\omega_{\mathrm{ZO}}\left(\vec{q}_{\perp}\right)=\omega_{\mathrm{ZO}}^{(0)}-c_{\mathrm{ZO}}\left|\vec{q}_{\perp}\right|\left(1-\frac{\left|\vec{q}_{\perp}\right|}{2 \sqrt{\pi \eta}}\right),
\end{gathered}
$$

where $c_{\mathrm{LO}}=\frac{\pi\left(e_{\mathrm{B}}^{\frac{1}{\mathrm{~B}}}\right)^{2}}{v_{2 \mathrm{D}} \mu \omega_{\mathrm{LO}}^{(0)}}$ and $c_{\mathrm{ZO}}=\frac{\pi\left(e_{\mathrm{B}}\right)^{2}}{v_{2 \mathrm{D}} \mu \omega_{\mathrm{ZO}}^{0}}$. Here, the frequencies $\omega_{\mathrm{LO}}$ and $\omega_{\mathrm{TO}}$ are degenerate at $\Gamma$, with symmetry $E^{\prime}$. The quantities $c_{\mathrm{LO}}$ and $c_{\mathrm{ZO}}$ have the dimension of velocities. We see that $\omega_{\mathrm{LO}}$ increases and $\omega_{\mathrm{ZO}}$ decreases linearly with $\left|\vec{q}_{\perp}\right|$ near $\Gamma$, on the other hand $\omega_{\mathrm{TO}}$ is flat. Further away from $\Gamma$, the quadratic decrease of $\omega_{\mathrm{LO}}$ with $\left|\vec{q}_{\perp}\right|$ is an indication of overbending, however overbending is already present in absence of Coulomb interactions [see Fig. 3(a)]. As already discussed in Sec. II, the HREELS experiments on a monolayer h-BN on $\mathrm{Ni}(111)$ do exhibit the degeneracy of the LO and TO modes at $\Gamma$. Although the LO mode increases with increasing wave vector near $\Gamma$, the experiments ${ }^{11}$ do not allow to identify a linear increase. However we would like to remind that the linear slope is a weak effect (since the charge transfer from $\mathrm{B}$ to $\mathrm{N}$ is relatively small) and possibly below the then available experimental resolution.

The density of states of the modes $\mathrm{LO}$ and $\mathrm{ZO}$ near the $\Gamma$ point is given by ${ }^{44}$

$$
g_{s}(\omega)=\frac{1}{(2 \pi)^{2}} \int \frac{d S_{\vec{q}_{\perp}}}{\left|\vec{\nabla} \omega_{s}\left(\vec{q}_{\perp}\right)\right|},
$$

where the index $s$ refers to LO and ZO. In Eq. (60) we have taken into account that the Brillouin zone is two-dimensional, $d S_{\vec{q}_{\perp}}=\left|\vec{q}_{\perp}\right| d \phi$ is the "surface element," i.e., the arc sustained by the angular element $d \phi$. Using $\left|\vec{\nabla} \omega_{s}\left(\vec{q}_{\perp}\right)\right|=c_{s}$ and integrating over the polar angle, we obtain

$$
g_{s}(\omega)=\frac{1}{2 \pi} \frac{\left|\vec{q}_{\perp}\right|}{c_{s}},
$$

and hence by means of Eq. (57)

$$
\begin{gathered}
g_{\mathrm{LO}}(\omega)=\frac{\omega-\omega_{\mathrm{LO}}^{(0)}}{2 \pi c_{\mathrm{LO}}^{2}}, \quad \omega>\omega_{\mathrm{LO}}^{(0)}, \\
g_{\mathrm{LO}}(\omega)=0, \quad \omega \leq \omega_{\mathrm{LO}}^{(0)},
\end{gathered}
$$

and by means of Eq. (59)

$$
\begin{gathered}
g_{\mathrm{ZO}}(\omega)=\frac{\omega_{\mathrm{ZO}}^{(0)}-\omega}{2 \pi c_{\mathrm{ZO}}^{2}}, \quad \omega<\omega_{\mathrm{ZO}}^{(0)}, \\
g_{\mathrm{ZO}}(\omega)=0, \quad \omega \geq \omega_{\mathrm{ZO}}^{(0)} .
\end{gathered}
$$

Experimental knowledge of the density of states $g_{\mathrm{LO}}$ and $g_{\text {ZO }}$ would lead to a determination of the effective charges $e_{B}^{\perp}$ and $e_{B}^{\|}$, respectively.

The group velocity of the LO mode still depends on the direction of the wave vector, with

$$
v_{i}^{\mathrm{LO}}\left(\vec{q}_{\perp}\right)=\frac{\partial \omega_{\mathrm{LO}}\left(\vec{q}_{\perp}\right)}{\partial q_{i}}=c_{\mathrm{LO}} \frac{q_{i}}{\left|\vec{q}_{\perp}\right|},
$$

$i \in\{1,2\}$. The slope $c_{\mathrm{LO}}$, calculated with the model parameters of Sec. III, has the numerical value $1.94 \mathrm{~km} / \mathrm{s}$. As a consequence we expect that the LO optical phonons contribute to the heat current and hence to thermal conductivity.

\section{CONCLUDING REMARKS}

We have given a theoretical lattice dynamical treatment of 2D h-BN which is based on a crystal model with partially covalent and ionic bonding. The calculated phonon spectra [Fig. 3(b)] are reasonably close to the in-plane IXS measurements of phonon dispersions in 3D h-BN (Ref. 13) and to the results of $a b$ initio calculations. ${ }^{13,22}$ However our main purpose has been the analytic implementation of Born's longwavelength method to the case of a noncentrosymmetric $2 \mathrm{D}$ ionic crystal. Since the crystalline structure of $2 \mathrm{D}$ h-BN with two atoms per unit cell is the most simple, it was possible to derive transparent analytic expressions for various physical quantities such as elastic tension coefficients and piezoelectric stress coefficients. In particular the important role of the linear term $i q_{k} D_{i j, k}^{(1) \mathrm{BN}}$ in the long-wavelength expansion of the dynamical matrix [Eq. (12)] has been elucidated. It has been shown how this term leads to a coupling of acoustic and optical phonons. The resulting corrections to the elastic tension coefficients $\gamma_{11}$ and $\gamma_{66}$ [round bracket terms in Eqs. (28) and (34)] are found to be quantitatively important. The elastic phenomena are mainly determined by the covalent part of the dynamical matrix, the Coulomb contribution is quantitatively small. As is to be expected, the evolution of the optical phonon branches, a.o. crossing of LO and TO modes, is rather sensitive to the choice of effective charges. Our restriction to the case of a rigid-ion model is possibly the reason of discrepancies with the LO-TO crossings observed in $a b$ initio calculations. ${ }^{13,22}$ In any case additional experi- 
mental results on 2D h-BN are needed to clarify the situation. Notwithstanding these shortcomings, we have demonstrated by rigorous calculations that the LO and TO modes are degenerate at the $\Gamma$ point. In addition the nonanalytic term of the Coulomb part of the dynamical matrix in two dimensions leads to a linear increase with $\vec{q}_{\perp}$ near $\Gamma$ of the LO branch and hence to an enhancement of overbending. In addition we find a decrease of the $\mathrm{ZO}$ branch as a function of the wave vector near $\Gamma$, while the TO branch remains flat. These findings are in agreement with $a b$ initio calculations where an enhancement of overbending by Coulomb interactions has been found as well. ${ }^{22}$ We have calculated the densities of states of the LO and ZO modes in the linear $\vec{q}_{\perp}$ regime near $\Gamma$ and have suggested measurements of the effective charges. Finally we have calculated the piezoelectric stress coefficient with the result $e_{1,11}=-1.19 \times 10^{-12} \mathrm{C} / \mathrm{cm}$. Extrapolation to a $3 \mathrm{D}$ crystal leads to a large value. We have found that in $2 \mathrm{D} \mathrm{h}-\mathrm{BN}$ the sound velocities and hence the tension coefficients are not changed by piezoelectric effects in the long-wavelength limit. This is a consequence of the vanishing of the long-wavelength Coulomb part of the dynamical matrix. The elastic sum rule which was originally derived for a nonionic and nonprimitive crystal remains valid. We conclude that from an elastic point of view a $2 \mathrm{D}$ ionic crystal behaves as a nonionic crystal. The situation is different for the optical modes where the Coulomb term leads to a linear $\left|\vec{q}_{\perp}\right|$ dependence. Our preliminary calculations on multilayer systems indicate that the slopes of the branches of the optical phonon band increase with the number of layers. This problem is currently under investigation.

\section{ACKNOWLEDGMENTS}

The authors acknowledge helpful comments from F. M. Peeters, A. Bosak, and M. Mohr. This work has been supported by the Flemish Science Foundation (FWO-Vl) and the Bijzonder Onderzoeksfonds, Universiteit Antwerpen (BOFUA).
${ }^{1}$ K. S. Novoselov, D. Jian, F. Schedin, T. J. Booth, V. V. Khotkevich, S. V. Morozov, and A. K. Geim, Proc. Natl. Acad. Sci. U.S.A. 102, 10451 (2005).

${ }^{2}$ A. K. Geim and K. S. Novoselov, Nature Mater. 6, 183 (2007).

${ }^{3}$ C. Jin, F. Lin, K. Suenaga, and S. Iijima, Phys. Rev. Lett. 102, 195505 (2009).

${ }^{4}$ K. Watanabe, T. Taniguchi, and H. Kanda, Nature Mater. 3, 404 (2004).

${ }^{5}$ R. Saito, G. Dresselhaus, and M. S. Dresselhaus, Physical Properties of Carbon Nanotubes (Imperial College Press, London, 1998).

${ }^{6}$ Understanding Carbon Nanotubes, edited by A. Loiseau, P. Launois, P. Petit, S. Roche, and J.-P. Salvetat (Springer Verlag, Berlin, Heidelberg, 2006).

${ }^{7}$ S. Reich, C. Thomsen, and J. Maultzsch, Carbon Nanotubes (Wiley-VCH, Weinheim, 2003).

${ }^{8}$ M. Mohr, J. Maultzsch, E. Dobardzic, S. Reich, I. Milosevic, M. Damnjanovic, A. Bosak, M. Krisch, and C. Thomsen, Phys. Rev. B 76, 035439 (2007).

${ }^{9}$ K. H. Michel and B. Verberck, Phys. Rev. B 78, 085424 (2008).

${ }^{10}$ A. Bosak, M. Krisch, M. Mohr, J. Maultzsch, and C. Thomsen, Phys. Rev. B 75, 153408 (2007).

${ }^{11}$ E. Rokuta, Y. Hasegawa, K. Suzuki, Y. Gamou, C. Oshima, and A. Nagashima, Phys. Rev. Lett. 79, 4609 (1997).

${ }^{12}$ A. Bosak, J. Serrano, M. Krisch, K. Watanabe, T. Taniguchi, and H. Kanda, Phys. Rev. B 73, 041402(R) (2006).

${ }^{13}$ J. Serrano, A. Bosak, R. Arenal, M. Krisch, K. Watanabe, T. Taniguchi, H. Kanda, A. Rubio, and L. Wirtz, Phys. Rev. Lett. 98, 095503 (2007).

${ }^{14}$ N. G. Chopra, R. J. Luyken, K. Cherrey, V. H. Crespi, M. L. Cohen, S. G. Louie, and A. Zettl, Science 269, 966 (1995).

${ }^{15}$ A. Loiseau, F. Willaime, N. Demoncy, G. Hug, and H. Pascard, Phys. Rev. Lett. 76, 4737 (1996).

${ }^{16}$ M. Born and K. Huang, Dynamical Theory of Crystal Lattices (Oxford University Press, Oxford, 1954).

${ }^{17}$ A. A. Maradudin, E. W. Montroll, G. H. Weiss, and I. Ipatova, in
Theory of Lattice Dynamics in the Harmonic Approximation, edited by H. Ehrenreich, F. Seitz, and D. Turnbull, Solid State Physics Suppl. 3 (Academic Press, New York, 1971).

${ }^{18}$ G. Kern, G. Kresse, and J. Hafner, Phys. Rev. B 59, 8551 (1999).

${ }^{19}$ N. Ohba, K. Miwa, N. Nagasako, and A. Fukumoto, Phys. Rev. B 63, 115207 (2001).

${ }^{20}$ T. Tohei, A. Kuwabara, F. Oba, and I. Tanaka, Phys. Rev. B 73, 064304 (2006).

${ }^{21}$ Y. Miyamoto, M. L. Cohen, and S. G. Louie, Phys. Rev. B 52, 14971 (1995).

${ }^{22}$ D. Sanchez-Portal and E. Hernandez, Phys. Rev. B 66, 235415 (2002).

${ }^{23}$ W. J. Yu, W. M. Lau, S. P. Chan, Z. F. Liu, and Q. Q. Zheng, Phys. Rev. B 67, 014108 (2003).

${ }^{24}$ V. N. Popov, Phys. Rev. B 67, 085408 (2003).

${ }^{25}$ L. Bonsall and A. A. Maradudin, Phys. Rev. B 15, 1959 (1977).

${ }^{26}$ M. H. Cohen and F. Keffer, Phys. Rev. 99, 1128 (1955).

${ }^{27}$ R. H. Lyddane and K. F. Herzfeld, Phys. Rev. 54, 846 (1938); H. Fröhlich and N. F. Mott, Proc. Roy. Soc. A 171, 496 (1939); R. H. Lyddane, R. G. Sachs, and E. Teller, Phys. Rev. B 59, 673 (1941).

${ }^{28}$ M. Topsakal, E. Aktürk, and S. Ciraci, Phys. Rev. B 79, 115442 (2009).

${ }^{29}$ R. Geick, C. H. Perry, and G. Rupprecht, Phys. Rev. 146, 543 (1966).

${ }^{30}$ T. Kuzuba, Y. Sato, S. Yamaoka, and K. Era, Phys. Rev. B 18, 4440 (1978).

${ }^{31}$ R. J. Nemanich, S. A. Solin, and R. M. Martin, Phys. Rev. B 23, 6348 (1981).

${ }^{32}$ P. M. Chaikin and T. C. Lubensky, Principles of Condensed Matter Physics (Cambridge University Press, Cambridge, England, 1995).

${ }^{33}$ L. D. Landau and E. M. Lifshitz, Elastizitätstheorie (Akademie Verlag, Berlin, 1987).

${ }^{34}$ I. Naumov, A. M. Bratkovsky, and V. Ranjan, Phys. Rev. Lett. 
102, 217601 (2009).

${ }^{35}$ R. Bechmann, Phys. Rev. 110, 1060 (1958).

${ }^{36}$ W. Götze and K. H. Michel, Z. Phys. 223, 199 (1969).

${ }^{37}$ L. D. Landau and E. M. Lifshitz, Elektrodynamik der kontinuierlichen Medien (Akademie Verlag, Berlin, 1987).

${ }^{38}$ W. Götze and K. H. Michel, Z. Phys. 217, 170 (1968).

${ }^{39}$ J. Maultzsch, S. Reich, C. Thomsen, H. Requardt, and P. Ordejón, Phys. Rev. Lett. 92, 075501 (2004).

${ }^{40}$ P. Pavone, R. Bauer, K. Karch, O. Schütt, S. Vent, W. Windl, D.
Strauch, S. Baroni, and S. de Gironcoli, Physica B 219-220, 439 (1996).

${ }^{41}$ C. Thomsen and S. Reich, Phys. Rev. Lett. 85, 5214 (2000).

${ }^{42}$ O. Dubay and G. Kresse, Phys. Rev. B 67, 035401 (2003).

${ }^{43}$ S. Piscanec, M. Lazzeri, F. Mauri, A. C. Ferrari, and J. Robertson, Phys. Rev. Lett. 93, 185503 (2004).

${ }^{44}$ L. D. Landau and L. M. Lifshitz, Statistische Physik Teil 1 (Akademie Verlag, Berlin, 1987). 